\title{
Epigenetic modification of Rta (ORF50) promoter is not responsible for distinct reactivation patterns of murine gammaherpesviruses
}

\author{
B. LAPUNÍKOVÁ ${ }^{1}$, K. LOPUŠNÁ2 ${ }^{2}$ T. BENKÓCZKA², F. GOLAIS ${ }^{1}$, M. KÚDELOVÁ2, I. REŽUCHOVÁ2
}

\begin{abstract}
${ }^{1}$ Department of Microbiology and Virology, Comenius University in Bratislava, Slovak Republic; ${ }^{2}$ Department of Molecular Pathogenesis of Viruses, Institute of Virology, Slovak Academy of Sciences, Dúbravská cesta 9, 84505 Bratislava, Slovak Republic.
\end{abstract}

Received May 21, 2015; revised July 22, 2015; accepted October 01, 2015

\begin{abstract}
Summary. - Gammaherpesviruses-encoded replication and transcription activator (Rta) (ORF50) plays an essential role in the initiation of viral lytic gene expression and reactivation from latency. The Rta expression is influenced by many viral and cellular factors, including epigenetic modifications, mainly DNA methylation and histone modifications. Murine gammaherpesvirus 68 (MHV-68), belonging to the species Murid herpesvirus (MuHV-4), is widely used as a model to study human gammaherpesvirus infections in vitro as well as in vivo. Recent studies of the MHV-68 Rta promoter revealed the effect of DNA demethylation and histone acetylation, induced by the inhibitor of histone deacetylase trichostatin A (TSA), on the MHV-68 reactivation from latency. Two other strains of MuHV-4, MHV-72 and MHV-4556, possess several unique properties, which distinguish them from strain MHV-68. Recently discovered reduced capacity of MHV-72 and MHV-4556 to reactivate from latency may be related to different methylation/demethylation patterns of the promoter regulatory region of the Rta. Here, we investigated the epigenetic regulation of the Rta promoter of three murine gammaherpesvirus strains, MHV-68, MHV-72 and MHV-4556, during latency and reactivation in vivo. However, we did not find any differences between Rta of MHV-68, MHV-72 and MHV-4556 and its epigenetic regulation during lytic infection, latency and de novo infection after ex vivo and in vivo reactivation induced by TSA. We confirmed that the treatment with TSA successfully induced demethylation of the Rta promoter regions of all three studied strains. Moreover, we have shown that the primary sequence of Rta and its promoter is identical for all three strains.
\end{abstract}

Keywords: murine gammaherpesvirus; trichostatin A; epigenetic modification; histone acetylation; gammaherpesvirus reactivation; replication and transcription activator

\section{Introduction}

Members of the subfamily Gammaherpesvirinae are relatively widespread viruses that can infect a variety of mammalian species, including humans. Human gam-

*Corresponding author. E-mail: viruorav@savba.sk; phone: +4212-59302460.

Abbreviations: $\mathrm{BGS}=$ bisulfite sequencing analysis; $\mathrm{CO}$ $\mathrm{BRA}=$ Combined bisulfite restriction analysis; d.p.i. = days post infection; EBV = Epstein-Barr virus; HDAC = histone deacetylases; KSHV = Kaposi's sarcoma associated herpesvirus; MHV$68=$ murine gammaherpesvirus $68 ; \mathrm{ORF}=$ open reading frame; Rta $=$ replication and transcription activator; TSA $=$ trichostatin $\mathrm{A}$ maherpesviruses, Epstein-Barr virus (EBV, HHV-4) and Kaposi's sarcoma associated herpesvirus (KSHV, HHV-8) are oncogenic viruses that induce a readily controlled lytic infection followed by the establishment of life-long latency (Pagano, 1999; Sarid et al., 1999). In most cases the persistent infection is asymptomatic or accompanied by benign cellular proliferation. However, persistent/chronic gammaherpesvirus infection is occasionally associated with the development of malignancies, such as Burkitt's lymphoma, Hodgkin's disease, and nasopharyngeal carcinoma as a result of EBV infection, and Kaposi's sarcoma, primary effusion lymphoma, and Castleman's disease after KSHV infection (Barton et al., 2011). Most of our knowledge of latent or acute infection with human gammaherpesviruses 
have been derived from in vitro studies. However, this approach does not allow revealing a whole complex of virus/ host interactions which take place during in vivo infection.

Murine gammaherpesvirus 68 (MHV-68), a natural pathogen of murid rodents belonging to the species Murid herpesvirus 4 (MuHV-4), is widely used as a model to study human gammaherpesviruses, answering recent aspects of virus infection and reactivation from latency, including a chronic infection which is associated with risk of lymphoproliferative disorders and oncogenesis. Together eight murine gammaherpesviruses designated $-60,-68,-72,-76$, $-78,-4556,-5682$, and -Šumava were isolated in Slovakia and thereafter in the Czech Republic (Blaškovič et al., 1980; Kožuch et al., 1993). Among them, the best-characterized isolate is MHV-68 (Nash et al., 2001). Despite the fact that the full genome sequence of MHV-72 and MHV-4556 is not known yet, some in vitro and in vivo pathogenetic studies revealed their close relation to MHV-68. However, several differences were observed among these three strains. It was shown that chronic infection with MHV-72 results in higher incidence of lymphomas in liver, lung and adrenals, which rises to nearly three-fold in mice treated with immunosuppressive drug compared to MHV-68 (Mistríková et al., 1996, 1999). Moreover, replication of MHV-72 in mammary glands and its transmission from infected mother mouse to neonates via breast milk was described. Further differences were revealed in studies focused on characterization of genome restriction map, nucleotide sequences of some unique genes including thymidine kinase, M3 protein, MK3 protein and glycoprotein 150, and some biological properties of MHV72 and MHV-4556 (Mačáková et al., 2003; Valovičová et al., 2006; Belvončíková et al., 2008; Halásová et al., 2011; Kúdelová et al., 2012). Recent results obtained in our study on biological properties of MHV-72 and MHV-4556 in vivo showed reduced ability of these strains to reactivate ex vivo from latency in spleen, lungs and thymus treated with trichostatin A (TSA), compared to MHV-68 (Lopušná et al., unpublished data).

All gammaherpesviruses encode a replication and transcription activator (Rta) homologue, which plays an essential role in the initiation of viral lytic gene expression and thus reactivation from latency. Two multifunctional proteins in EBV, known as ZEBRA (BZLF1) and Rta (BRLF1), and the one in KSHV, known as Rta (ORF50), are responsible for regulation of latent/lytic switch. The Rta encoded by gene ORF50 of gammaherpesviruses shares similarities in genomic location, sequence, and splicing pattern, confirming that they play conserved and important role in virus life cycle (Quinlivan et al., 1993; Ragoczy et al., 1998; Ragoczy and Miller, 2001; Russo et al., 1996; Whitehouse et al., 1997).

MHV-68 Rta is responsible for transactivation of both its own promoter and promoters of other viral and cellular genes, resulting in reactivation of latent virus and lytic rep- lication (Hair et al., 2007). It has been previously shown that an Rta null mutant is incapable of viral protein synthesis, viral DNA replication or virion production. In addition to the transactivation of viral promoters by Rta, the reactivation as well as the phase of viral dormancy are influenced by several cellular factors, including the epigenetic modifications, which have been extensively studied in past ten years. Epigenetic modifications, as changes in gene activity and expression that occur without alteration in DNA sequence, are tightly regulated by two major epigenetic modifications: DNA methylation and histone modifications (Goldberg et al., 2007; Bernstein et al., 2007).

Previous studies have shown that both DNA methylation and histone acetylation regulate EBV and KSHV reactivation from latency (Chen et al., 2001; Lu et al., 2003; Chang and Liu, 2000; Countryman et al., 2008; Ben-Sasson and Klein, 1981). Recently, Yang et al. (2009) investigated the effect of DNA demethylation, mediated by methyltransferase inhibitor 5-aza-2-deoxycytidine (5-AzaC), and histone acetylation, induced by the inhibitor of histone deacetylases trichostatin $\mathrm{A}$, on the $\mathrm{MHV}-68$ reactivation from latency. For the effective reactivation of MHV-68 from latency only the histone acetylation induced by TSA was needed. In addition, they identified 15 methylation sites (CpG) localised in the MHV-68 Rta promoter. Further analyses confirmed differential methylation patterns of Rta promoter in virion, during lytic infection of $\mathrm{BHK}-21$ cells, during latency in S11E cell line transformed by MHV-68 and finally during reactivation induced by 5 -aza-2-deoxycytidine and TSA in vitro and in vivo.

Despite the intensive study, the role of epigenetic regulation in MHV-68, MHV-72 and/or MHV-4556 reactivation in vivo has not been reported yet. We suppose that unique properties of MHV-72 and MHV-4556, mainly in the context of reduced capacity to reactivate from latency, may be related to different methylation/demethylation pattern of the promoter regulatory region of the transactivator protein Rta. In this study, we investigated the epigenetic regulation of the Rta promoter of three murine gammaherpesvirus strains, MHV-68, MHV-72 and MHV-4556 during latency and reactivation.

\section{Materials and Methods}

Cells and viruses. BHK-21[C-13] (baby hamster kidney fibroblast cell line, ATCC ${ }^{\oplus}$ CCL-10 ${ }^{\mathrm{TM}}$ ) and S11E (murine B-cell line transformed with MHV-68 obtained from prof. J.P. Stewart, PhD., Department of Veterinary Pathology, The University of Edinburgh, United Kingdom) (Usherwood et al., 1996) cells were cultured in Dulbecco's modified Eagle's medium (DMEM) supplemented with $10 \%$ fetal bovine serum (HyClone), $2 \mathrm{mmol} / \mathrm{l} \mathrm{L}$-glutamine, $100 \mathrm{U}$ of streptomycin per $\mathrm{ml}$ and $100 \mathrm{U}$ of penicillin per $\mathrm{ml}$ (Lonza). 
Experiments were performed with the gammaherpesviruses MHV-68 clone f2.6, MHV-72 clone h3.7 (Rašlová et al., 2000) and MHV-4556 clone i2.8 (Valovičová et al., 2006). Working virus stocks were prepared after infection of BHK-21 cells at the multiplicity of infection (MOI) of $0.05 \mathrm{PFU} /$ cell.

Animals. Six-week-old BALB/c mice were obtained from Laboratory Animal Breeding and Experimental Facility of Faculty of Medicine, Masaryk University in Brno, Czech Republic. Mice were bred and housed in a specific pathogen-free barriers facility in accordance with institutional and state guidelines (Ro 3497/14-221). The mice were infected intranasally with $2 \times 10^{5} \mathrm{PFU}$ of virus in 25 $\mu \mathrm{l}$ of DMEM following an Isoflurane anesthesia. For ex vivo reactivation, mice were sacrificed with an Isoflurane overdose followed by cervical dislocation at 14 and 35 days post infection (d.p.i.). Spleens were collected and cultured in DMEM supplemented with TSA (200 ng/ml) for 10 days followed by DNA isolation. For in vivo reactivation, mice ( 35 d.p.i.) were stimulated with TSA (2.4 $\mathrm{mg} / \mathrm{kg}$ ) subcutaneously for $5 \mathrm{hr}$ and subsequently sacrificed with an Isoflurane overdose followed by cervical dislocation. Control mice were handled with the same manner as infected mice with the exception of TSA treatment. Non-infected mice served as a negative control. Spleens were harvested and rapid frozen in liquid nitrogen $\left(-195.79^{\circ} \mathrm{C}\right)$ prior to the DNA isolation.

DNA isolation. DNA from S11E cells, infected BHK-21 cells, and spleen after ex vivo and in vivo reactivation was extracted using the QIAamp DNA mini kit (Qiagen) according to the instructions of the manufacturer. DNA was stored at $-20^{\circ} \mathrm{C}$ until further use.

Bisulfite conversion, COBRA, methylation specific PCR and cloning. Bisulfite conversion of DNA (up to $600 \mathrm{ng}$ ) was performed using the EpiJET kit for bisulfite conversion (Thermo Scientific) according to the instructions of the manufacturer. Converted as well as non-converted DNA was analyzed by Combined bisulfite restriction analysis (COBRA) - cleavage combination of methylation specific restriction enzymes MspI/HpaII (Thermo Scientific). Rta promoter region, which was divided into two fragments, P1 (65695-66258nt) and P2 (66355-66879nt), was analyzed for methylation status during latency and in vivo induced reactivation of MHV-68, MHV-72 and MHV-4556 by methylation specific PCR. Bisulfide converted DNA from spleen of infected mice was amplified by methylation specific and non-specific primers (Table 1). The amplified fragments were purified by Wizard ${ }^{\oplus} S V$ gel and PCR clean-up system (Promega) according to the instructions of the manufacturer. The amplicons were cloned into the pJET1.2 blunt cloning vector system (Thermo Scientific) according to the instructions of the manufacturer and analyzed by sequencing. Obtained sequences were compared with the reference sequence of MHV-68 (Acc. No. U 97553).

Sequencing. For analysis of primary structure of the genomic region corresponding to the ORF50 (66760-69373nt) and the Rta promoter region (65644-66800nt) of MHV-72 and MHV-4556 we used conventional PCR with primers listed in Table 1. All fragments were cloned into the pJET1.2 blunt cloning vector system (Thermo Scientific) and analyzed by sequencing. The obtained sequences were compared with the MHV-68 sequence.

\section{Results and Discussion}

MHV-68 encodes an immediate-early replication transactivator protein Rta (ORF50) in a similar genomic location to other gammaherpesviruses. Moreover, all gammaherpesvirus Rta transcripts share a similar architecture. They comprise two exons separated by an intron, which is essentially composed of the ORF49 gene (Fig. 1a,b,c). Splicing of the two exons and

Table 1. List of primers

\begin{tabular}{|c|c|c|c|}
\hline Name of primer & Sequence (5'- 3') & Genomic location & Notes \\
\hline CPORF50/ F1 & CAAGGACCATGGCAACTAGTCTC & $65643-65665$ & used for sequencing \\
\hline CPORF50/R2 & CTACCTTATCGGCTGAAGGGG & $66799-66779$ & used for sequencing \\
\hline CPORF50R3 & TAGCACATCTGTTGTGTGGGC & $66936-66916$ & used for sequencing \\
\hline $50 \mathrm{WF} 1^{\star 1}$ & GGCCGCAGACATTTAATGAC & 68147-68166 & used for sequencing \\
\hline $50 \mathrm{WR} 1^{* 1}$ & AACTGGAACTCTTCTGTGGC & $68733-68714$ & used for sequencing \\
\hline ORF50MHVF1 & CAAAGTCCATAACAGGCATCC & $67939-67959$ & used for sequencing \\
\hline ORF50MHVR1 & CTGTGGGAGAAGCAAACAGG & $68450-68431$ & used for sequencing \\
\hline YTHHP1-F*2 & GGTTTTTGTGTAGAATTTTTGATTATGA & $65695-65722$ & used for BGS \\
\hline YTHHP1-R ${ }^{* 2}$ & ССААССТСАССААСТТТТАСААТА & $66258-66235$ & used for BGS \\
\hline YTHHP2-F*2 & TTTTTTGAATAGAGTGAGAAGGGTAG & $66355-66380$ & used for BGS \\
\hline YTHHP2-R ${ }^{* 2}$ & TCAAACTAATAACAACACTTTAATTTTTAA & $66879-66858$ & used for BGS \\
\hline YTHHP1-M-F*2 & TGTTGGTTACGTTTAGGTATTCGA & 65791-65814 & methylation specific, used for BGS \\
\hline YTHHP1-M-R ${ }^{* 2}$ & ATCTCACTAAAAACACTCCAACGAC & $66084-66060$ & methylation specific, used for BGS \\
\hline YTHHP2-M-F*2 & GTATTACGAGGGAATTTTTGTAGC & $66753-66776$ & methylation specific, used for BGS \\
\hline YTHHP2-M-R ${ }^{* 2}$ & ATTTTTAATAAAATACTAATCTATCTACGT & $66857-66828$ & methylation specific, used for BGS \\
\hline
\end{tabular}

${ }^{*}$ Weck et al. (1999); ${ }^{* 2}$ Yang et al. (2009). 


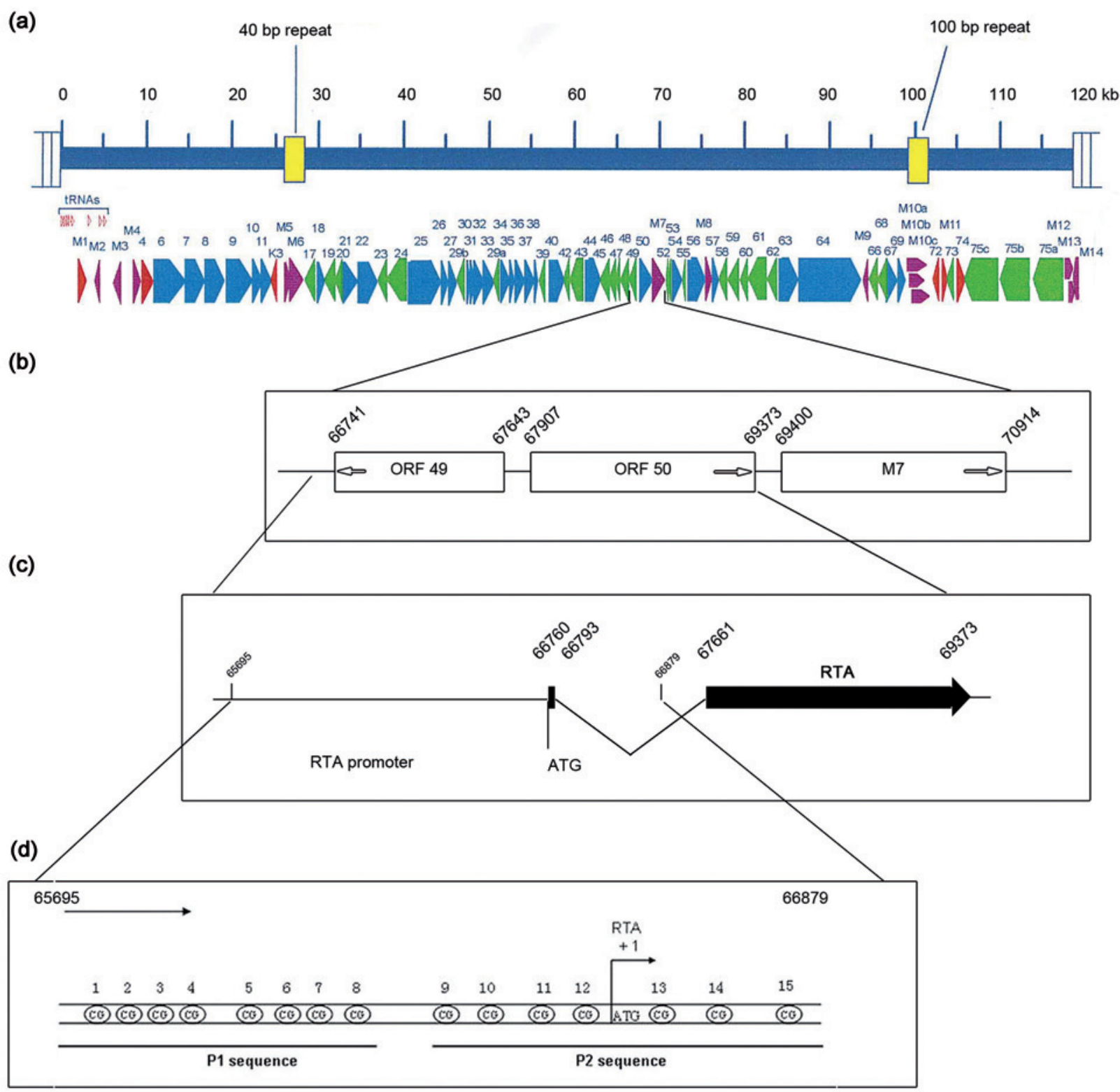

Fig. 1

Schematic diagram of the Rta/ORF50 MHV-68

(a) Schematic diagram of the MHV-68 genome and genomic location of ORF50. The nucleotide numbers are assigned on the basis of the MHV-68 sequence (Acc. No. U97553) published in the National Center for Biotechnology Information database (by Virgin et al., 1997). (b) Detail view of the ORF49, ORF50 and M7 genomic region. (c) Scheme of Rta transcription region. (d) Detail view of the Rta promoter region (by Yang et al., 2009).

excision of the intervening intron results in a single, major Rta transcript, but also many bicistronic alternatively spliced Rta-containing transcripts can be presented in infected cell. Despite the fact, that ORF50 genes of gammaherpesviruses are highly conserved, the amino acid similarity between KSHV and rhesus monkey rhadinovirus (RRV) Rta is 55\%, between KSHV and MHV-68 Rta is 43\%, and between RRV and MHV-68 Rta is 47\% (Damania et al., 2004). However, these differences do not affect the function of Rta protein. Therefore, we considered the possibility that murine gammaherpesvirus strains could possess the amino acid diversity in Rta protein. In present study we identified the nucleotide sequence of the ORF50 gene (66760-69373nt) and its proximal promoter region (65695 -66879nt) of MHV-72 and MHV-4556 (Fig. 1c).

Sequences obtained from pJET1.2 recombinants were assembled into a contiguous sequence representing the 
region of the ORF50 and its promoter. Short sequence, approximately $640 \mathrm{nt}$, on the 3 ' end, which could not be covered by any combination of primers, was verified by the sequencing of the whole genomes of MHV-72 and MHV-4556 by Next-generation sequencing (data not published yet). The sequence analysis showed that the nucleotide sequences of ORF50 gene and its proximal promoter region of MHV-72 and MHV-4556 are identical to that of MHV-68.

However, the regulation of gene expression not only depends on nucleotide sequence of promoter in conjunction with activators and suppressors of gene expression but is also spatially and temporally regulated by epigenetic modifications. These epigenetic mechanisms include DNA methylation, remodelling of nucleosomes, expression of small regulatory RNAs and posttranslational modifications of histone proteins (Bird, 2007). Relatively well characterized chromatin modifications, DNA methylation and histone acetylation, are important for gene transcription regulation, cell development, and also tumorigenesis (Feinberg and Tycko, 2004; Esteller, 2008; Jones and Baylin, 2007). DNA methylation occurs at the 5 position of the cytosine ring within $\mathrm{CpG}$ dinucleotides via addition of a methyl group to create a 5-methylcytosine. This process is catalysed by several DNA methyltransferases in eukaryotic cells. Acetylation of histones promotes gene transcription by relaxing chromatin structure and facilitating access of the transcription machinery to DNA, whereas histone deacetylation promotes transcriptional repression by condensation of chromatin structure (Van Opdenbosch et al., 2012). To date, 18 cellular histone deacetylases (HDACs) providing deacetylation of histones are known. Their negative regulators, inhibitors of HDACs, inhibit deacetylation of histones resulting in histones acetylation and thus gene transcription (Damania et al., 2004).

Histones associated with virus genome are important for establishment and maintaining a latent circular viral episome during gammaherpesvirus latency. In addition, transcription from episomal DNA is epigenetically regulated and the gene expression is silenced except for a small number of genes responsible for the maintenance of the episome in the host cells (Eshleman et al., 2011; Djerbi et al., 1999; Ballestas et al., 1999). In previous experiments it was shown that HDAC3 complex is recruited to the Rta promoter of MHV- 68 and HDACs 1, 5, and 7 are recruited to the Rta promoter of KSHV to suppress gene transcription during latency. However, after treatment with HDAC inhibitors, acetylated histones can be found on gammaherpesvirus lytic switch gene promoters (Goodwin et al., 2010). An example can be the treatment with TSA, leading to the removal of HDAC3 from the Rta promoter, up-regulation of histone $\mathrm{H} 3$ and $\mathrm{H} 4$ acetylation level and MHV-68 reactivation. Reactivation is accompanied by passive demethylation at the Rta promoter. These findings indicate that histone acetylation induced by TSA, but not

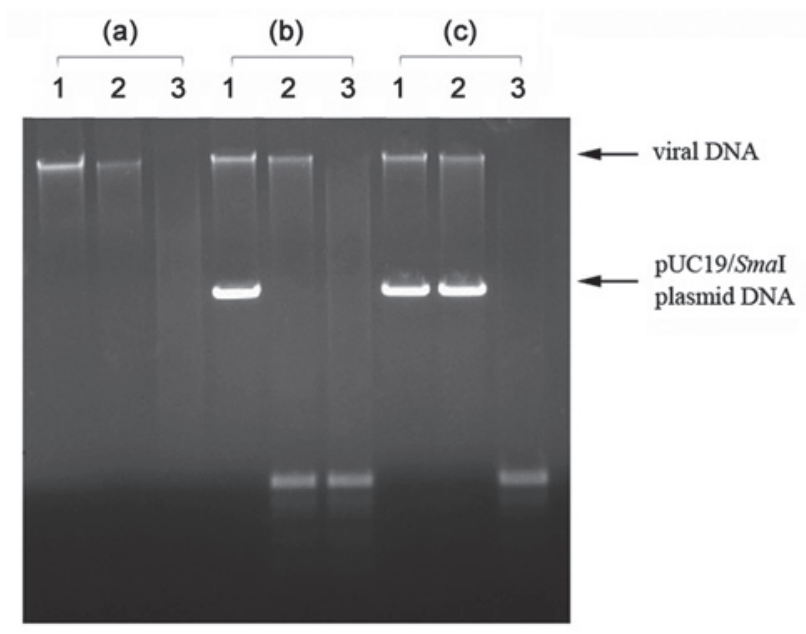

Fig. 2

COBRA analysis of MHV-72 DNA methylation status during late latency (35 d.p.i.) in spleen

(a) MHV-72 DNA from spleen of mice (35 d.p.i.): 1 - non-digested, 2 - digested with HpaII, 3 - digested with MspI; (b) MHV-72 DNA from spleen of mice (35 d.p.i.) mixed with non-methylated pUC19/SmaI plasmid DNA: 1 - non-digested, 2 - digested with HpaII, 3 -digested with MspI; (c) MHV-72 DNA from spleen of mice (35 d.p.i.) mixed with methylated pUC19/SmaI plasmid DNA: 1 - non-digested, 2 - digested with HpaII, 3 - digested with MspI.

DNA demethylation, is sufficient for efficient reactivation of MHV-68 from latency (Yang et al., 2009).

These facts lead us to hypothesise that the strain-dependent reactivation ability (Lopušná et al., unpublished data) may be associated with distinct epigenetic modification of Rta promoter, including its methylation status and susceptibility on demethylation enzymes, although we identified that the nucleotide sequences of the ORF50 and its promoter are identical for all three strains tested - MHV-68, MHV-72 and MHV-4556.

We investigated the methylation status of MHV-72 and MHV-4556 DNA during different stages of viral life cycle in vitro and in vivo. For COBRA analysis we used: 1 . DNA isolated from BHK-21 cells infected with MHV-4556 (48 hours post infection) - lytic replication in vitro; 2 . DNA isolated from the spleen of mice at 14 d.p.i. with MHV-4556 and MHV-72 - early latency in vivo; 3. DNA isolated from the spleen of mice at 35 d.p.i. with MHV-4556 and MHV-72 - late latency in vivo; 4 . DNA isolated from the spleen of mice at 35 d.p.i. with MHV-4556 and MHV-72, after ex vivo reactivation induced by TSA - ex vivo reactivation; 5 . DNA isolated from $\mathrm{S} 11 \mathrm{E}$ cell line - natural reactivation. In this cell line MHV-68 occurs in the latent state with a low spontaneous reactivation frequency. We used The Thermo Scientific ${ }^{\mathrm{TM}}$ EpiJET $^{\mathrm{TM}}$ DNA methylation analysis kit (MspI/HpaII), with a pair of restriction enzymes with different sensitivity to 


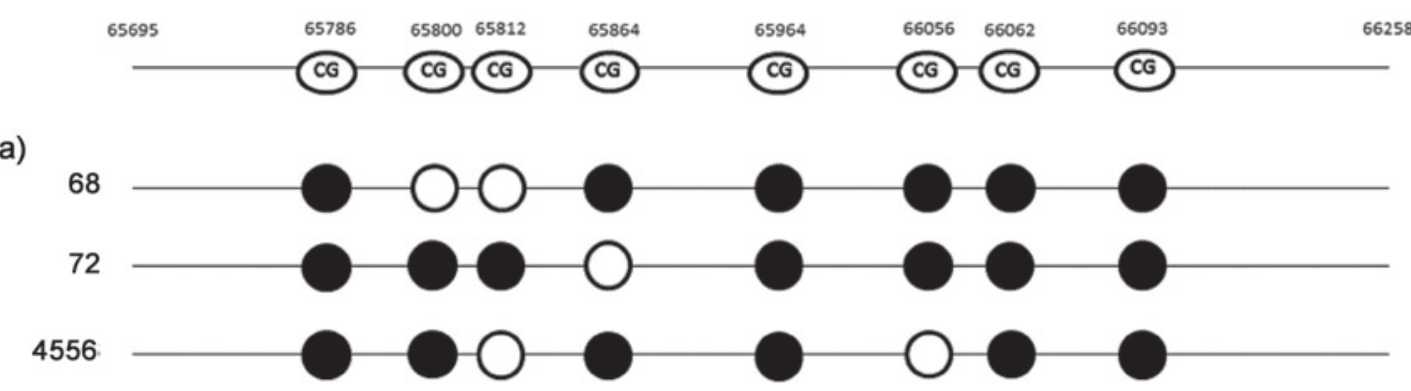

(b)

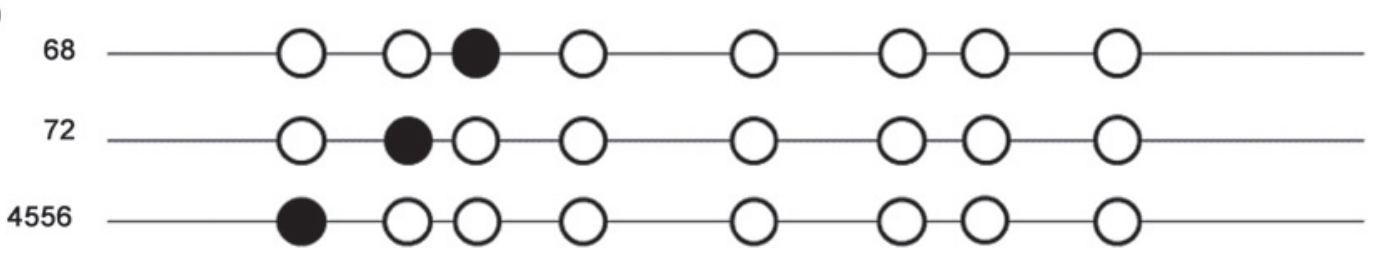

Fig. 3

Bisulfite genomic sequencing (BGS) analysis of the CpG sites at the distal region of Rta promoter of MHV-68, MHV-72 and MHV-4556 The distal region (65695-66258nt) of the Rta promoter and localization of $8 \mathrm{CpG}$ sites in it. (a) Methylation status of the CpG sites during late latency of MHV-68, MHV-72 and MHV-4556 in spleen at 35 d.p.i. (b) Methylation status of the CpG sites during in vivo reactivation of MHV-68, MHV-72 and MHV-4556 induced by TSA $(2.4 \mathrm{mg} / \mathrm{kg})$ in spleen at 35 d.p.i. Solid circles indicate methylated CpG, open circles indicate demethylated CpG.

methylated DNA. When the CpG site is methylated, DNA cleavage with HpaII is blocked, although the cleavage with MspI is not affected.

We have found that the DNA isolated from BHK-21 cells infected with MHV-4556 (1) could be digested by HpaII, suggesting that DNA is mostly unmethylated during lytic replication in vitro (48 hours post infection). DNA isolated from spleen of mice infected with MHV-4556 and MHV-72 was resistant to HpaII digestion (Fig. 2, line a2, b2, c2), indicating mostly methylated status of DNA during the early latency (2) and the late latency in spleen (3). During the ex vivo reactivation induced by TSA (4) and also during the natural reactivation in S11E cell line (5), the DNA showed the presence of both, methylated and unmethylated cytosines, since it was partially resistant to HpaII digestion (data not shown).

With respect to the fact, that the ex vivo induced reactivation does not include the complexity of in vivo processes and virus/host interactions, we investigated virus reactivation from latency in vivo, which was induced by TSA administered as a subcutaneous injection to mice at 35 d.p.i. with MHV-68, MHV-72 or MHV-4556. For detailed examination of the methylation/demethylation status of MHV-4556 and MHV-72 Rta promoters during latency and after in vivo induced reactivation, we performed the bisulfite sequencing analysis (BGS) consisting of the bisulfite conversion of unmethylated cytosines, the methylation specific PCR (MSP) and the sequencing of amplified Rta fragments. The 1kbp MHV-68, MHV-72, and MHV-4556 Rta promoter region was divided into two fragments, defined as $\mathrm{P} 1$ and P2, which include a total of $15 \mathrm{CpG}$ sites (Fig. 1d). The distal region of Rta promoter, the fragment P1 (65695-66258 nt), consists of $8 \mathrm{CpG}$ sites, the proximal region, the fragment $\mathrm{P} 2$ (66355-66879 nt), consists of 7 CpG sites, which surround the transcription start of the Rta $(66760 \mathrm{nt})$. We found that during latency (35 d.p.i.), most of the CpG sites in the P1 fragment of Rta promoter were methylated (Fig. 3a) and all of the CpG sites in the P2 fragment were methylated (data not shown). However, no significant differences of methylation status of Rta promoter region between the strain MHV-68, MHV-72 and MHV-4556 were detected in late latency in vivo. TSA treatment during in vivo induced reactivation (for $5 \mathrm{hr}$ ) led to the effective demethylation of most of the CpG sites in the P1 fragment of Rta promoter (Fig. 3b) and all CpG sites in the P2 fragment (data not shown).

Taken together, these data indicate that the methylation patterns of the Rta promoter of MHV-68, MHV-72 and MHV4556 were remarkably uniform during latency as well as the demethylation patterns of the Rta promoter of all three strains during in vivo reactivation induced by TSA. Furthermore, we can conclude that the sensitivity of MHV-72 and MHV-4556 to HDAC inhibitor Trichostatin A is similar within all three strains, MHV-68, MHV-72 and MHV-4556.

First evaluation of the Rta methylation status associated with MHV-68 infection in vitro and in vivo was done by Yang et al. (2009). Similarly to our results, they found that the viral Rta 
promoter region of MHV-68 was highly methylated during latency in vitro and in vivo, whereas the Rta promoter was mostly unmethylated during de novo, in vitro or in vivo infection.

Summarizing, we did not find any differences between studied murine gammaherpesvirus strains - MHV-68, MHV-72 and MHV-4556, which could be associated with the replication transcription activator, Rta (ORF50), and its epigenetic regulation during lytic infection, latency and also de novo infection after ex vivo and in vivo induced reactivation. Moreover, we confirmed that the treatment with TSA successfully induced demethylation of the Rta promoter regions of all three studied strains, and this occurred by passive mechanism (Yang et al., 2009).

Therefore, the unique in vivo pathogenetic properties of MHV-72 and MHV-4556, including mainly the reduced capacity to reactivate from latency followed by low amounts of genome copies of de novo assembled virus, could be related to another gene or genes, in which they differ from prototype strain MHV-68.

Acknowledgement. This research was supported by the Slovak Research and Development Agency (\#APVV-0621-12) and the joint grant agency of Slovak Ministry of Education and Slovak Academy of Sciences VEGA ( $\# 2 / 0185 / 12)$.

\section{References}

Belvončíková $\mathrm{P}$, Králová $\mathrm{A}$, Kúdelová $\mathrm{M}$, Režuchová $\mathrm{I}$, Vančová I (2008): Chemokine-binding activities of M3 protein encoded by Murine gammaherpesvirus 72. Acta Virol. 52, 91-97.

Ballestas ME, Chatis PA, Kaye KM (1999): Efficient persistence of extrachromosomal KSHV DNA mediated by latencyassociated nuclear antigen. Science 284, 641-644. http:// dx.doi.org/10.1126/science.284.5414.641

Barton E, Mandal P, Speck S (2011): Pathogenesis and host control of gammaherpesviruses: Lessons from the mouse. Annu. Rev. Immunol. 29, 351-397. http://dx.doi.org/10.1146/ annurev-immunol-072710-081639

Ben-Sasson SA, Klein G (1981): Activation of the Epstein-Barr virus genome by 5 -aza-cytidine in latently infected human lymphoid lines. Int. J. Cancer 28, 131-135. http://dx.doi. org/10.1002/ijc.2910280204

Blaškovič D, Stančeková M, Svobodová J, Mistríková J (1980): Isolation of five strains of herpesviruses from two species of free living small rodents. Acta Virol. 24, 468.

Bernstein BE, Meissner A, Lander ES (2007): The mammalian epigenome. Cell 128, 669-681. http://dx.doi.org/10.1016/j. cell.2007.01.033

Bird A (2007): Perceptions of epigenetics. Nature 447, 396-398. http://dx.doi.org/10.1038/nature05913

Chang LK, Liu ST (2000): Activation of the BRLF1 promoter and lytic cycle of Epstein-Barr virus by histone acetylation. Nucleic Acids Res. 28, 3918-3925. http://dx.doi. org/10.1093/nar/28.20.3918
Chen J, Ueda K, Sakakibara S, Okuno T, Parravicini C, Corbellino M, Yamanishi K (2001): Activation of latent Kaposi's sarcoma-associated herpesvirus by demethylation of the promoter of the lytic transactivator. Proc. Natl. Acad. Sci. USA 98, 4119-4124. http://dx.doi.org/10.1073/ pnas. 051004198

Countryman JK, Gradoville L, Miller G (2008): Histone hyperacetylation occurs on promoters of lytic cycle regulatory genes in Epstein-Barr virus-infected cell lines which are refractory to disruption of latency by histone deacetylase inhibitors. J. Virol. 82, 4706-4719. http://dx.doi. org/10.1128/JVI.00116-08

Damania B, Jeong JH, Bowser BS, DeWire SM, Staudt MR, Dittmer DP (2004): Comparison of the Rta/Orf50 transactivator proteins of gamma-2-herpesviruses. J. Virol. 78, 5491-5499. http://dx.doi.org/10.1128/JVI.78.10.5491$\underline{5499.2004}$

Djerbi M, Screpanti V, Catrina AI, Bogen B, Biberfeld P, Grandien A (1999): The inhibitor of death receptor signaling, FLICE-inhibitory protein defines a new class of tumor progression factors. J. Exp. Med. 190, 1025-1032. http:// dx.doi.org/10.1084/jem.190.7.1025

Eshleman E, Shahzad A, Cohrs RJ (2011): Varicella zoster virus latency. Future Virol. 6, 341-355. http://dx.doi.org/10.2217/ $\underline{\text { fvl.10.90 }}$

Esteller M (2008): Epigenetics in cancer. N. Engl. J. Med. 358, 1148-1159. http://dx.doi.org/10.1056/NEJMra072067

Feinberg AP, Tycko B (2004): The history of cancer epigenetics. Nat. Rev. Cancer. 4, 143-153. http://dx.doi.org/10.1038/ $\underline{\operatorname{nrc1279}}$

Goldberg AD, Allis CD, Bernstein E (2007): Epigenetics: a landscape takes shape. Cell 128, 635-638. http://dx.doi. org/10.1016/j.cell.2007.02.006

Goodwin MM, Molleston JM, Canny S, El Hassan MA, Willert EK, Bremner R, Virgin HW (2010): Histone deacetylases and the nuclear receptor corepressor regulate lytic-latent switch gene 50 in murine gammaherpesvirus 68 -infected macrophages. J. Virol. 84, 12039-12047. http://dx.doi. org/10.1128/JVI.00396-10

Hair JR, Lyons PA, Smith KG, Efstathiou S (2007): Control of Rta expression critically determines transcription of viral and cellular genes following gammaherpesvirus infection. J. Gen. Virol. 88, 1689-1697. http://dx.doi.org/10.1099/ vir.0.82548-0

Halásová Z, Valovičová M, Mačáková K, Pančík P, Belvončíková P, Režuchová I, Kúdelová M (2011): Partial genome sequence of murine gammaherpesvirus 72 and its analysis. Acta Virol. 55, 317-325. http://dx.doi.org/10.4149/ av $2011 \quad 04 \quad 317$

Jones PA, Baylin SB (2007): The epigenomics of cancer. Cell 128, 683-692. http://dx.doi.org/10.1016/j.cell.2007.01.029

Kožuch $O$, Reichel M, Leššo J, Remeňová $A$, Labuda M, Lysý J, Mistríková J (1993): Further isolation of murine herpesviruses from small mammals in southwestern Slovakia. Acta Virol. 37, 101-105.

Kúdelová M, Halásová Z, Belvončíková P, Pančík P, Režuchová I, Valovičová M (2012): Partial genome analysis of murine 
gammaherpesvirus 4556. Acta Virol. 56, 177-186. http:// dx.doi.org/10.4149/av 2012 $03 \quad 177$

Lu F, Zhou J, Wiedmer A, Madden K, Yuan Y, Lieberman PM (2003): Chromatin remodeling of the Kaposi's sarcomaassociated herpesvirus ORF50 promoter correlates with reactivation from latency. J. Virol. 77, 11425-11435. http://dx.doi.org/10.1128/JVI.77.21.11425-11435.2003

Mačáková K, Matis J, Reýuchová I, Kúdela $\mathrm{O}$, Rašlová $\mathrm{H}$, Kúdelová M (2003): Murine gammaherpesvirus (MHV) M7 gene encoding glycoprotein 150 (gp150): difference in the sequence between 72 and 68 strains. Virus Genes 26, 89-95. http://dx.doi.org/10.1023/A:1022390407991

Mistríkova J, Rajčáni J, Mrmusová M, Oravcová I (1996): Chronic infection of Balb/c mice with murine herpesvirus 72 is associated with neoplasm development. Acta Virol. 40, 297-301.

Mistríková J, Mrmusová M, Ďurmanová V, Rajčáni J (1999): Increased neoplasm development due to immunosuppressive treatment with FK-506 in BALB/C mice persistently infected with the mouse herpesvirus (MHV-72). Viral Immunol. 12, 237-247. http://dx.doi.org/10.1089/ vim.1999.12.237

Nash AA, Dutia BM, Stewart JP, Davison AJ (2001): Natural history of murine gamma-herpesvirus infection. Philos Trans. R Soc. Lond. B Biol. Sci. 356, 569-579. http://dx.doi. org/10.1098/rstb.2000.0779

Pagano JS (1999): Epstein-Barr virus: the first human tumor virus and its role in cancer. Proc. Assoc. Am. Physicians. 111, 573-580. http://dx.doi.org/10.1046/j.1525-1381.1999. t01-1-99220.x

Quinlivan EB, Holley-Guthrie EA, Norris M, Gutsch D, Bachenheimer SL, Kenney SC (1993): Direct BRLF1 binding is required for cooperative BZLF1/BRLF1 activation of the Epstein-Barr virus early promoter, BMRF1. Nucleic Acids Res. 21, 1999-2007. http://dx.doi.org/10.1093/ $\underline{\text { nar/21.8.1999 }}$

Ragoczy T, Heston L, Miller G (1998): The Epstein-Barr virus Rta protein activates lytic cycle genes and can disrupt latency in B lymphocytes. J. Virol. 72, 7978-7984.

Ragoczy T, Miller G (2001): Autostimulation of the Epstein-Barr virus BRLF1 promoter is mediated through consensus Sp1 and Sp3 binding sites. J. Virol. 75, 5240-5251. http:// dx.doi.org/10.1128/JVI.75.11.5240-5251.2001
Rašlova H, Mistrikova J, Kudelova M, Mishal Z, Sarasin A, Blangy D, Berebbi M (2000): Immunophenotypic study of atypical lymphocytes generated in peripheral blood and spleen of nude mice after MHV-72 infection. Viral Immunol. 13, 313-327. http://dx.doi. org $/ 10.1089 / 08828240050144644$

Russo JJ, Bohenzky RA, Chien MC, Chen J, Yan M, Maddalena D, Parry JP, Peruzzi D, Edelman IS, Chang Y, Moore PS (1996): Nucleotide sequence of the Kaposi sarcomaassociated herpesvirus (HHV8). Proc. Natl. Acad. Sci. USA 93, 14862-14867. http://dx.doi.org/10.1073/ pnas.93.25.14862

Sarid R, Olsen SJ, Moore PS (1999): Kaposi's sarcoma-associated herpesvirus: epidemiology, virology, and molecular biology. Adv. Virus Res. 52, 139-232. http://dx.doi. org/10.1016/S0065-3527(08)60299-7

Usherwood EJ, Stewart JP, Nash AA (1996): Characterization of tumor cell lines derived from murine gammaherpesvirus68-infected mice. J. Virol. 70, 6516-6518.

Valovičová M, Režuchová I, Mačáková K, Petrová P, Matis J, Kúdelová M (2006): Murine gammaherpesvirus (MHV) MK3 gene sequence diversity among 72, 4556, and 68 strains. Virus Genes 33, 51-58. http://dx.doi.org/10.1007/ s11262-005-0038-4

Van Opdenbosch N, Favoreel H, Van de Walle GR (2012): Histone modifications in herpesvirus infections. Biol. Cell 104, 139-164. http://dx.doi.org/10.1111/boc.201100067

Virgin HW 4th, Latreille P, Wamsley P, Hallsworth K, Weck KE, Dal Canto AJ, Speck SH (1997): Complete sequence and genomic analysis of murine gammaherpesvirus 68. J. Virol. 71, 5894-5904.

Weck KE, Kim SS, Virgin HW IV, Speck SH (1999): Macrophages are the major reservoir of latent murine gammaherpesvirus 68 in peritoneal cells. J. Virol. 73, 3273-3283.

Whitehouse A, Carr IM, Griffiths JC, Meredith DM (1997): The herpesvirus saimiri ORF50 gene, encoding a transcriptional activator homologous to the Epstein-Barr virus $\mathrm{R}$ protein, is transcribed from two distinct promoters of different temporal phases. J. Virol. 71, 2550-2554.

Yang Z, Tang H, Huang H, Deng H (2009): RTA promoter demethylation and histone acetylation regulation of murine gammaherpesvirus 68 reactivation. PLoS One 4, e4556. http://dx.doi.org/10.1371/journal.pone.0004556 